\title{
Entre pornographie et politique. La fabrication de l'ethos de puissance dans le discours pornographique externe : le cas des biographies érotiques d'acteurs $X$ homo- et bisexuels en ligne
}

\author{
Between Pornography and Politics. The Construction of the Power \\ Ethos in External Pornographic Discourse: the Case of Erotic \\ Biographies of Gay and Bisexual Porn Actors Online
}

Mateusz Białas

Université de Białystok, Pologne

Résumé : Le langage de la pornographie se déploie aujourd'hui non seulement dans les dictionnaires des mots du sexe ou dans les textes, mais aussi sur diverses pages Web. Notre étude se fonde sur un corpus pour lequel nous avons analysé un matériau variationnel relevant du non-standard : une centaine de biographies érotiques d'acteurs $X$ homo- et bisexuels rassemblées sur le site francophone https://www.videosxgays.com/. L'objectif de notre analyse est de nous pencher sur la construction discursive de l'identité des acteurs $X$ pour mettre en avant l'ethos de puissance qui se dégage dans le discours étudié à travers un répertoire spécifique d'instruments langagiers.

Mots-clés : acteur, biographie, bisexualité, discours, ethos, homosexualité, pornographie.

Abstract: Today the language of pornography unfolds not only in sex word dictionaries or texts, but also on various web pages. Our study is based on a corpus for which we analyzed a variational material relating to the non-standard: around a hundred erotic biographies of homo- and bisexual pornstars gathered on the French-speaking site https://www.videosxgays.com/. The objective of our analysis is to look at the discursive construction of the identity of $X$ actors in order to highlight the power ethos that emerges in the discourse studied through a specific repertoire of linguistic instruments.

Keywords: actor, biography, bisexuality, discourse, ethos, homosexuality, pornography. 


\section{Introduction}

Le discours social n'est pas un phénomène homogène; bien au contraire, il est possible d'en mettre en avant plusieurs types, comme par exemple les discours institutionnel, médiatique, religieux, politique, publicitaire, scientifique, juridique, journalistique, etc. Qui plus est, il semble fort intéressant de voir que les discours, et surtout l'ensemble des discours sociaux, ne sont pas, comme le souligne Marie-Anne Paveau (2014, p. 25), " seulement des suites de mots et de phrases, mais constituent des lieux où se définissent les normes et les valeurs, les prescriptions et les interdits, les goûts et les dégoûts, les qualités et les défauts, les identités, les légitimités, les gloires et les hontes. Sous cet angle, les univers discursifs pornographiques constituent de passionnants observatoires de la vie sociale, car ils sont au croisement de plusieurs grands domaines qui l'organisent: la sexualité, bien sûr, entraînant avec elle les normes des désirs et des plaisirs, la morale, qui n'a cessé à travers les âges de prendre l'exemple de la pornographie et du sexe en général pour définir ses propres valeurs, mais également la forme de nos relations intimes, notre rapport au sexe et au genre, les dimensions politiques de nos pratiques sexuelles, l'intrication de la sexualité et de la technique ॥. Or, il importe de souligner également que dans la sphère médiatique la plus large, comme le laisse entendre François-Ronan Dubois (2014, p. 4), auteur du premier ouvrage de synthèse francophone sur les études pornographiques (angl. porn studies) cité par Paveau (2014, p. 230), on se penche souvent sur la pornographie et " on y parle (...) comme d'une chose générale, mais on n'y parle guère des documents pornographiques $»$.

\section{Corpus et cadre théorique}

Néanmoins, il n'est pas difficile de noter que le langage de la pornographie se déploie aujourd'hui non seulement dans les dictionnaires des mots du sexe ou dans les textes, par exemple les textes médiatiques, polémiques, littéraires, etc., mais aussi sur les blogs et les pages Web. Parmi de tels sites Internet, il existe des portails spécifiques, à savoir les pages à contenus pornographiques, appelées également des pages X. Leur popularité et, par conséquent, l'efficacité ne se restreint pas uniquement à leur côté visuel bien que c'est l'image qui y joue un rôle capital. Ceci à plus forte raison parce que le côté verbal de ces pages - aussi modeste et accessoire soit-il par rapport à leur côté iconique - paraît jouer ainsi un rôle à ne pas sous-estimer. Notre étude se fonde sur un corpus pour lequel nous avons analysé un matériau variationnel diastratique relevant du non-standard: une centaine de documents écrits qui représentent les fiches d'information (ca. 8500 mots) voire les biographies érotiques de 93 acteurs de l'amour charnel, en l'occurrence celles des acteurs $X$ homo- et bisexuels rassemblées sur le site francophone: https://www.videosxgays.com/. Ces dernières constituent, quant à elles, un exemple frappant du genre de discours pornographique externe - l'un des trois phénomènes distingués et caractérisés par Paveau (2014, p. 48). Selon la chercheuse, le discours pornographique englobe:

- le discours sur la pornographie : celui de l'évaluation qui peut avoir pour fonctions la défense, la critique voire une stigmatisation violente des formes de représentation hors les normes ;

- le discours interne : celui qui relève de la production ou de l'œuvre pornographique, à savoir le contenu discursif de l'œuvre littéraire, du script, de la bande dessinée, de l'œuvre d'art, etc. ; 
- le discours externe : celvi qui émane de l'édition ou l'industrie pornographique et qui a principalement pour but de nommer, de classer, de catégoriser des œuvres, des produits, etc. en vue d'attirer le plus grand nombre possible de récepteurs.

Observons donc de plus près quelques données quantitatives de notre analyse de cas concernant essentiellement le corps / le physique des acteurs X homo- et bisexuels ainsi que le graphique dévoilant la répartition des acteurs selon le pays d'origine :

- nombre des cas $=93$

- âge moyen $=31,1$ ans [20-50]

- taille moyenne $=177,5 \mathrm{~cm}$ [167-193]

- poids moyen $=74 \mathrm{~kg}$ [54-104]

- position :

$$
\begin{array}{ll}
\checkmark & \text { active }=25 \text { cas } \\
\checkmark & \text { passive }=7 \text { cas } \\
\checkmark & \text { polyvalente = } 20 \text { cas } \\
\checkmark & \text { nd. }=41 \text { cas }
\end{array}
$$

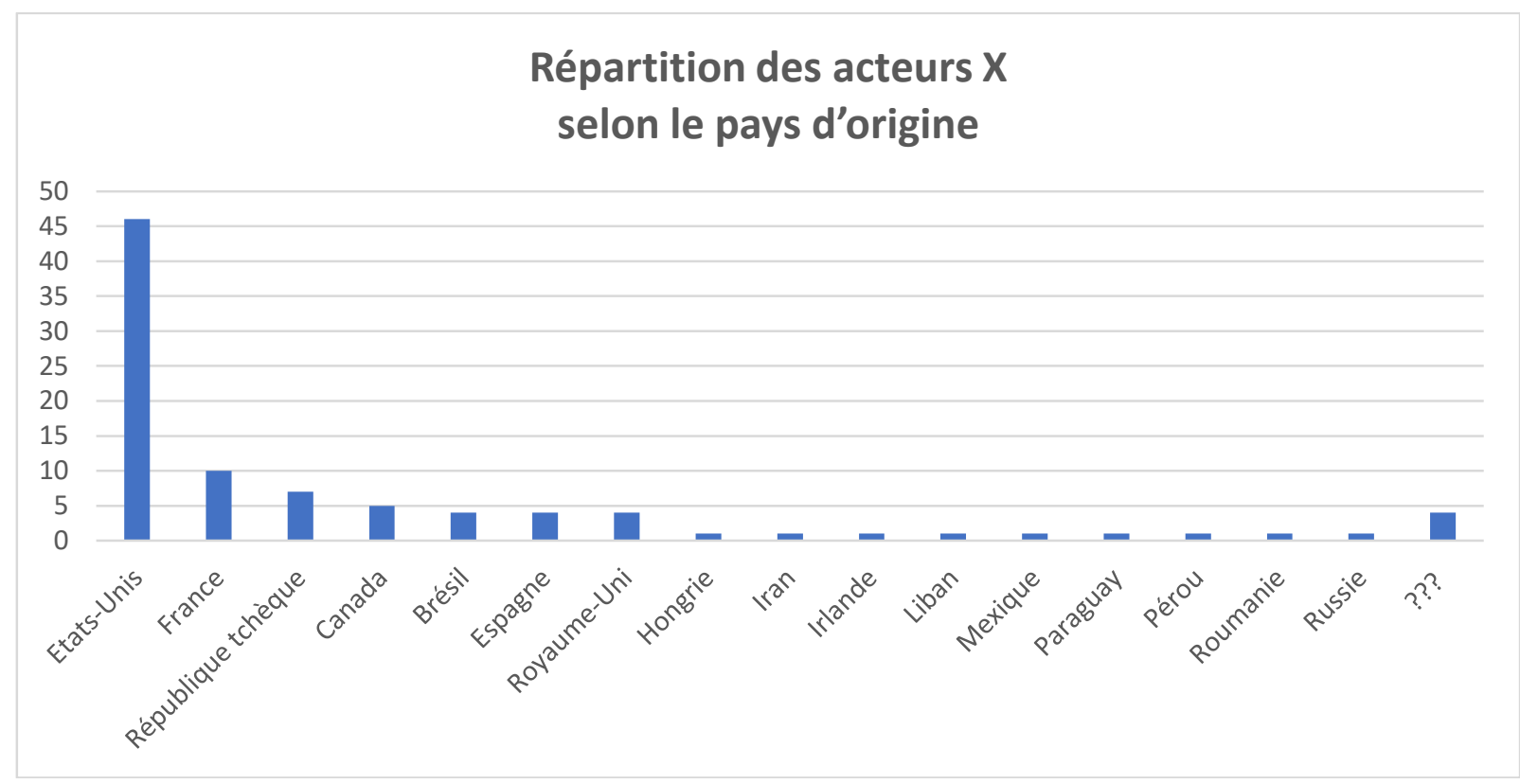

\section{Cadre conceptuel : contexte, méthodologie et objectif}

Pour commencer, il convient de faire remarquer que « croiser la problématique gaie avec la question du corps, c'est reconnaître d'emblée la centralité du corps dans la culture gaie. Eł de fait, elle peut être déclinée sur trois niveaux distincts, mais intimement liés $)$, à savoir une esthétique du corps, une éthique du corps et une politique du corps (Tin, 2018, pp. 234-236). De manière un peu taquine, on pourrait dire que le corps, lui aussi, peut jouer un rôle important dans le discours politique dont l'objectif fondamental est de persuader et / ou de séduire le destinataire tout en fabriquant un imaginaire bien particulier et complexe, composé souvent de maintes figures. Pourtant, en ce qui concerne le discours politique, on parle plutôt de la construction des ethos que des imaginaires, la catégorie provenant de la tradition lacaniennie où elle se résume à ce qui reflète de façon inconsciente le désir dans 
l'image que le sujet a de lui-même (Lacan, 1971-1972, p. 154). Les ethos, quant à eux, s'organisent autour de deux catégories majeures telles que les ethos de crédibilité et, avant tout, ceux d'identification « dont les images puisent (...) dans l'affect social : le citoyen, au travers d'un processus d'identification irrationnel, fond son identité dans celle de l'homme politique " (Charaudeau 2005, p. 105). C'est précisément ici que se novent - nous semble-t-il - des liens interdiscursifs très intéressants entre ces deux types de discours: politique et pornographique. En fait, ce dernier, comme le suggèrent Marie-Anne Paveau et François Perea (2014, p. 12), " est, sous de nombreux aspects, un discours politique, en particulier en ce qui concerne les rapports sociaux de sexe (pornographie féministe, alternatif, post-pornographie), et la dimension politique du corps et des émotions sensuelles (la sexualité publique comme performance militante par exemple) "I. L'analyse du discours que nous présentons dans cet article relève de I'hypothèse selon laquelle l'efficacité pragmatique du discours en question prend sa source dans la fabrication particulière de l'ethos (imaginaire), considéré comme l'une des caractéristiques génériques du discours politique hégémonique.

Ensuite, il faudrait observer que parmi les ethos d'identification dont nous venons de parler, il en existe un qui paraît frappant du point de vue de l'analyse linguistique du discours pornographique à travers les documents qui font partie du corpus recueilli, ceci à plus forte raison parce qu'il fait placer le corps humain à la position centrale. II s'agit de l'imaginaire de puissance qui est appréhendé comme une énergie physique, corporelle, qui " anime et propulse le corps dans l'action » (Charaudeau, 2005, p. 106). Cet ethos, contrairement à celui du pouvoir, est relatif non pas à l'organisation de la vie collective, mais à l'individu et « il nous renvoie l'image d'une force de la nature, force tellurique contre laquelle on ne peut pas grand-chose » (Charaudeau, 2005, p. 106). Contrairement encore au discours politique, surtout hégémonique où «ce sont toujours les gouvernants qui adressent leurs messages aux gouvernés" (Białas, 2020, p. 7), le discours pornographique ne devient le discours social que depuis les années quatre-vingts du XXe siècle. En effet, " (...) dès 1984 aux États-Unis, l'anthropologue et militante féministe Gayle Rubin (1984, p. 136) déclarait : « II est grand temps de parler du sexe ». (...) c'est-à-dire ouvrir un champ d'études en sciences humaines et sociales, mais aussi permettre, pour des raisons tant politiques et éthiques que sociales et culturelles, humanistes pour tout dire, que le sexe ne soit plus caché voire honteux dans la société étatsunienne comme ailleurs " (Paveau \& Perea 2014, p. 8).

Finalement, pour en revenir donc à la politique du corps, l'un des enjeux essentiels pour la construction de l'imaginaire identitaire, il convient de mettre en exergue le fait que «le corps homosexuel (...) cantonné à la sphère privée, sous le regard médical ou psychiatrique, (...) accède désormais à la sphère publique, et devient politique ॥ (Tin 2018, p. 36). Dans cette optique, nous trouvons très intéressant de voir que l'imaginaire de puissance peut se manifester dans le discours politique hégémonique à travers une figure bien spécifique: celle de virilité sexuelle (Charaudeau, 2005, p. 106). Or, pour ce qui est du discours pornographique externe examiné dans cet article, cette dernière est, selon nous, contrairement au discours politique hégémonique, toujours explicitement déclarée et se trouve ainsi au cœur de la construction de l'imaginaire identitaire dans les biographies érotiques étudiées. En effet, la fonction identitaire, primordiale parmi toutes les fonctions que peut remplir la variation linguistique (Kacprzak \& Goudaillier, 2014, p. 4), semble revêtir une 
manifestation particulière dans les biographies examinées. L'objectif de notre analyse est donc de nous pencher sur la construction discursive de l'identité des acteurs $X$ homo- et bisexuels pour mettre en avant l'imaginaire identitaire qui se dégage dans le discours étudié à travers un répertoire spécifique d'instruments langagiers. Pour ce qui est de ces derniers, il convient de signaler que l'analyse des procédés lexicorhétoriques dans le discours pornographique externe que nous proposons dans cet article prend pour point de départ méthodologique la classification des principales figures du discours de Jean-Jacques Robrieux (2000).

\section{Analyse de cas}

\subsection{Mec, mâle, étalon : identité sexuelle masculine}

Dans le discours analysé, les acteurs $X$ sont d'abord identifiés en tant que hommes et plus particulièrement comme homo sexualis. Mais comment sont-ils exactement ? En fait, il s'avère que l'image de l'homme qui se dégage à travers les biographies recueillies n'est pas tout à fait homogène, ce qui se reflète bien à travers le langage utilisé dans le discours pour désigner les acteurs $X$ masculins. Or, il convient de noter que l'homme tel qu'il est présenté dans les documents examinés se caractérise principalement par l'ensemble des qualités telles que la beauté physique (" ce beau mâle d'origine du Costa Rica vit aux Etats Unis... ») et la force musculaire (" ce beau mâle à la carrure sportive »).

A ces deux caractéristiques saillantes s' ajoutent d'autres traits distinctifs qui mettent en valeur la dimension biologique, voire animalière de l'homme tels que la vigueur redoutable (" sous ses allures de mâle alpha... " ; " une véritable bête sous les draps »), les instincts (" Un peu comme une seconde vie, quand il quitte sa tenue de cuisinier en province, l'étalon kabyle se transforme en... »; « (...) et quand on voit la bête en action, il est facile de le croire »), la reproduction (" Un bel étalon musclé, Perse, ancien militaire et également mannequin »), la fécondation (« Un bel étalon arabe $100 \%$ actif qui ne manquera pas de vous charmer $» ;$ " (...) mais tu ne t'imagineras sans doute pas quelle bête de sexe il devient quand il enlève ses vêtements »). Par-dessus tout, il importe de noter que c'est la puissance sexuelle qui semble caractériser les acteurs $X$ le plus manifestement. En font preuve quelques exemples saisissants tirés de notre corpus : « ce beau mâle viril de la vingtaine d'années »; « ce beau mâle aussi bien à l'aise en actif qu'en passif... ॥, etc.

Comme nous pouvons le voir dans les exemples évoqués ci-dessus, les trois noms qui apparaissent dans le corpus à maintes reprises (« mâle ") : 10 fois, "étalon " : 6 fois, "bête ": 3 fois) comme instruments lexicaux pour catégoriser les acteurs $X$ tout en renvoyant directement à l'aspect physique voire physiologique et animale de l'existence humaine, en l'occurrence masculine. En outre, il est pertinent d'observer que selon le Dictionnaire Larousse, seuls un mâle et une bête sont employés au registre autre que courant : le premier nom, au registre familier, signifie un homme vigoureux, moralement ou physiquement, en particulier caractérisé par la puissance sexuelle tandis que le second, au registre littéraire désigne l'homme sous l'emprise de ses instincts.

Toutefois, en sus de la beauté et de la force physiques qui sont mises en exergue de manière presque obsessionnelle dans les biographies examinées, il importe de souligner que l'imaginaire identitaire des acteurs X masculins s'appuie également sur d'autres valeurs explicitement déclarées, dont la jeunesse semble devenir une valeur fondamentale: «ce jeune mec sûr de lui "; «ce jeune mec est intelligent et 
sexy... » ; " un jeune mec bien foutu »; « un jeune mec avec un bon cul rebondi », etc. Aussi ressort-il de notre examen du corpus recueilli que c'est la prédilection envers l'amour charnel (« un excellent baiseur, viril, et diablement performant "; " ce baiseur chevronné saura combler tous ses partenaires "; " ce bogosse est un baiseur nu... ॥) aussi bien que le très grand pénis (" un mec viril avec une grosse... " ; " ce beau gosse actif avec un sexe de $20 \mathrm{~cm}$ »; " ce minet au corps fin et à la bite énorme représente à merveille le cliché du beau gosse français ») qui marquent le caractère distinctif des acteurs en question. En effet, les "mecs» dont l'âge moyen s'élève à 31 ans sont représentés dans le discours étudié comme de jeunes êtres humains du sexe masculin qui semblent aimer le coït de façon particulière voire démesurée, qui ont de l'expérience et qui sont compétents en la matière.

De plus, il est intéressant d'observer que ces trois noms (mec : 13 fois; bogosse : 6 fois; baiseur : 3 fois) apparaissent dans bien des dictionnaires comme par exemple Collins Dictionary en ligne, selon lequel ils sont bel et bien présents dans la langue française depuis le début du XVIIIe siècle. Dans le cas du beau gosse, il importe de voir aussi une modification volontaire de la chaîne écrite, typique du registre familier du français: en effet, le bogosse constitue quant à lui un exemple parlant d'un métaplasme par substitution. Pourtant, nous trouvons fascinant de faire remarquer que dans notre corpus, cette altération concerne aussi une inversion des syllabes à l'intérieur du substantif en question qui y apparaît sous une forme verlanisée : gossbo - un exemple frappant d'un métaplasme par permutation produisant le plus souvent un effet de drôlerie.

\subsection{Belle gueule, pur coquin, taureau sexuel : identité professionnelle}

Penchons-nous maintenant sur l'identité des acteurs créés par l'industrie pornographique, à savoir celle des stars et des célébrités du cinéma X. En fait, leur image publique est accessible pratiquement pour tout le monde - c'est là où l'on peut noter le caractère externe du discours pornographique. En effet, c'est un discours social par excellence qui s'approche du discours publicitaire : les acteurs y sont dépeints et valorisés comme des produits de luxe. On voit ici une immense force persuasive du discours - le langage doit attirer les récepteurs : on met en avant la singularité des acteurs $X$ qui semble prendre sa source non seulement dans leur beauté masculine exceptionnelle, mais aussi dans leurs capacités sexuelles extraordinaires.

Avant toute chose, il faudrait voir que cette singularité est accentuée dans les documents examinés par le biais des moyens langagiers (et rhétoriques) spécifiques; le cas échéant, il est question de figures du discours dont le pouvoir argumentatif semble incontestable. Ainsi l'analyse du corpus nous a-t-elle permis de mettre en avant deux types d'expressions qui font référence à l'identité professionnelle des acteurs X. D'une part, on peut déceler nombre de constructions valorisantes nom + épithète / épithète + nom dénotant le plus souvent leur beauté corporelle aussi bien que leurs qualités de métier exceptionnelles: « une belle gueule »; " un excellent partenaire »; " un pur coquin »; " un amant extraordinaire » ; " un taureau sexuel »; " ce baiseur chevronné »; " cet acteur exclusif », etc.

D'autre part, notre corpus abonde en appellations louangeuses ressemblant à des slogans publicitaires désignant les stars érotiques telles que les expressions 
métaphoriques, souvent en réduplication, (" la révélation de 2017 »; « la révélation de la fin d'année de men.com »; « la star internationale de l'année par Prowler en 2018 ») et les périphrases servant à ennoblir la réalité dépeinte dans le discours examiné (« la coqueluche des studios CockyBoys et Helix Studio "; " une recette de succès aux studios Bel Ami »). Or, il est indispensable de faire remarquer que le corpus étudié abonde principalement en hyperboles exagérant la réalité dans un sens laudatif, appelées auxèses. En voici quelques exemples évocateurs de cette figure du discours dévoilant l'identité toute singulière des vedettes de l'amour physique : "l'incarnation du mâle parfait ॥; "l'incarnation parfaite du minet américain »; "l'une des idoles pornos les plus réussies dans le monde entier "; " cette figure emblématique du studio Bel Ami ॥; " le mélange parfait de travail et de plaisir », etc.

\subsection{Viril, doux, sexy : identités psychologique et sociale}

Outre la figure de virilité sexuelle explicitement déclarée dans le discours qui fait objet de notre étude (" Un excellent baiseur, viril et diablement performant ॥; " II est toujours vigoureux et possède une tonne d'énergie sexuelle »), il importe de voir que la construction de l'imaginaire de puissance s'appuie sur des figures plus atténuées. En effet, l'image des acteurs $X$ semble s'adoucir par la valorisation de deux autres aspects de leur identité : psychologique et social.

Pour ce qui est de l'identité psychologique, il ressort de notre analyse que les stars masculines de l'amour charnel se caractérisent essentiellement par le charme attirant (" Ce beau mâle viril de la vingtaine d'années charme avec son style à mi chemin entre le méditerranéen et le latino... »; «Son charme et son pouvoir de séduction font craquer ses partenaires qui s'abandonnent à lui... " ; " Un espagnol bien d'entrain avec un charme fou à découvrir sur notre sélection de vidéos gratuites "; " Un homme charmant dans la rue et une véritable bête sous les draps... ॥), voire par le charisme inouï (" Nathan Hope est un jeune Parisien de la vingtaine avec beaucoup de charisme qui se lâche complètement dans ses scènes porno ॥; "Ce qu'on aime chez Iui ? Sa virilité, son corps de dieu et son charisme »).

Par surcroît, une autre constatation pertinente qui s'impose à la lumière de l'étude du corpus réuni est le fait que les acteurs se distinguent par deux autres traits psychologiques importants, à savoir la douceur (" Ariel a une personnalité très douce et très câline "; " C'est un mec viril avec un grosse queve épaisse et il a un côté doux et sensible ») et la sensualité (" Une belle gueule qui envoie du lourd, qui transpire la sensualité »; " A la fois juvénile et sexy »). Pourtant, il est indispensable d'observer que ces caractéristiques beaucoup plus psychologiques que physiques s'amalgament aux autres traits de caractère dont sont dotés les acteurs, comme par exemple l'intelligence (" Ce jeune mec est intelligent et sexy, calme et sûr de lui quand il s'agit de s'éclater »), la créativité (« II est une personne extrêmement créative avec un talent musical époustouflant »), la détermination à agir (" Le jeune et beau Jeffrey est déterminé ») et même la timidité (" En grandissant, il affirme avoir été très timide et ne même pas avoir pu se doucher ou se changer devant les autres gars dans le vestiaire 1). En bref, il importe de voir que l'homme tel qu'il est présenté dans le discours analysé est susceptible d'avoir une personnalité qui semble loin d'être simple. Qu'il suffise de prendre pour exemple une citation saillante provenant de notre corpus : « (...) mais sa personnalité ouverte et sa confiance en lui sont tellement excitants! Avec toutes les manières d'un paysan chevaleresque de l'Ohio, vous pouvez également dire que quelque chose de mauvais et, si j'ose dire, "arrogant" se cache sous le personnage ॥. 
Comme le montrent les exemples évoqués ci-dessus, le corpus analysé nous fournit bien des détails intéressants sur l'identité des acteurs - son aspect psychologique en particulier y est mis en avant par le biais d'une accumulation du vocabulaire à valeur essentiellement positive, souvent hyperbolique. En effet, il est indispensable de voir que c'est le côté verbal (et non pas visuel !) de la page Web examinée avec son lexique glorifiant qui "fait apprivoiser» les acteurs en tant que personnes vivantes avec une identité qui n'est pas réduite au corps. En d'autres termes, les instruments lexicaux mobilisés sont supposés créer l'avant de la rencontre sexuelle, tout en contribuant à fabriquer - pour reprendre les termes de l'analyse rhétorique (Amossy, 2006) - l'ethos pré-discursif des acteurs, c'est-à-dire l'image que le récepteur peut se construire des acteurs préalablement à leur mise en jeu, l'image qui ne le laissera pas indifférent.

D'ailleurs, c'est pour cela avant tout que nous avons décidé de parler de biographies érotiques et non pas pornographiques, la différence entre l'érotisme et la pornographie relevant du qualitatif et non du quantitatif. Selon Michela Marzano (2017), ce n'est pas le caractère explicite de l'image sexuelle qui la rend pornographique; or, comme le constate cette chercheuse en philosophie morale et politique, il faut faire remarquer que « la véritable différence est d'ordre qualitatif, car, là où l'érotisme est une représentation en mots ou en images de la rencontre sexuelle et de tout ce que cela implique en termes de peurs, d'attentes, de désirs, d'espoirs, de frustrations, de failles, etc., et la pornographie est une représentation qui prétend montrer l'acte sexuel en tant que tel, indépendamment de l'avant et de l'après, indépendamment des différences subjectives qui jovent toujours un rôle important dans une rencontre sexuelle. $)(2017$, p. 751).

Sans nous attarder sur des explications qui débordent de ce cadre, il faut souligner que les biographies analysées sont donc « plus » érotiques que pornographiques dans la mesure où elles dévoilent quelque chose de plus que seul l'acte sexuel, elles montrent de façon plus ou moins minutieuse l'avant de l'amour physique : toute la diversité des portraits des acteurs avec leur identité sociale qui englobe non seulement leurs origines et leurs professions (" Je suis un jeune ouvrier du Kentucky qui a toujours voulu tourner des films porno "; "Chanteur libanais, designer, activiste, entraîneur personnel et acteur porno " ; ll possède son diplôme universitaire en dentisterie et réside actuellement dans le quartier du sud de Manhattan à New York. Rafael a également travaillé comme mannequin. II a posé pour des créateurs aussi connus que Calvin Klein »), mais aussi la multitude de leurs talents, de leurs intérêts, de leurs passions, etc. En voici quelques citations intéressantes de notre corpus qui en témoignent: "Theo aime faire de l'équitation, aller à la gym et pratiquer les arts martiaux. Il est également un voyageur passionné, un intérêt suscité par son éducation multiculturelle qui mêle l'héritage français de son père à celui de sa mère irlandaise née au Maroc "; " Rafael parle 5 langues dont l'hébreu, l'allemand et le portugais »; " J'aime le cinéma, jouer de la musique, écrire et je joue au dodgeball dans une ligue de compétition depuis un peu moins de six ans et j'adore le camping $॥$.

\section{Conclusion}

Pour conclure, il s'ensuit à la lumière de notre étude du corpus réuni que le contexte culturel dans lequel se manifeste l'imaginaire identitaire de puissance, à savoir celui des pays occidentaux hautement médiatisés, repose sur un système de valeurs qui 
met en exergue l'esprit de conquête, et notamment celui de réussite. Dans le monde virtuel à l'heure actuelle, ce succès reflète principalement la popularité des acteurs qui s'exprime quant à elle par le nombre des vues et non pas celui des voix. Or, il convient de noter également que, contrairement au discours politique hégémonique, les moyens langagiers bien particuliers y sont employés dans l'intention de produire non pas un effet de repoussoir vis-à-vis de la star masculine, mais celui d'attrait, ce qui veut dire qu'ils ne sont mobilisés qu'en vue d'exalter et non pas de disqualifier les acteurs du cinéma $X$, comme ceci est souvent le cas des hommes politiques. En effet, l'ethos de puissance semble beaucoup plus masculin que féminin : l'engagement du corps en tant que preuve de vérité est glorifié à fond dans le discours pornographique examiné.

Comme nous pouvons l'observer, parmi les instruments langagiers dont se sert l'émetteur du discours analysé afin de mettre en relief l'imaginaire identitaire des acteurs fondé notamment sur une figure de virilité sexuelle, il en existe une véritable cascade des termes qui ont des connotations améliorantes extrêmement suggestives. Ce sont tantôt des métaphores affectueuses et / ou humoristiques constituant fréquemment des appellatifs hypocoristiques ("la coqueluche du studio "; "une boule d'énergie »; "une bombe du porno ॥; " cette bombe moka »; "la bête en action »; « un hardeur hors pair »), tantôt des périphrases qui renvoient à l'idéal du monde antique et médiéval, divin et chevaleresque ("cet adonis au corps sculpté »; " un dieu du sexe »; "un chevalier à l'armure étincelante »), tantôt des hyperboles valorisantes (" une usine à fantasme à lui seul "; « un véritable monstre du sexe »; "la véritable définition du mec brun ténébreux à la peau basanée »; "une représentation sensuelle parfaite de la beauté méditerranéenne $\|)$ et de nombreuses réduplications (" parfait », « incarnation », « mec », « bogosse ॥, " charme ॥, etc.).

En bref, nous tenons à souligner que

[...] si chaque année, la marche des fiertés lesbiennes, gaies, bi et transsexuelles apparaît d'abord comme un grand carnaval, une théorie de corps dénudés, une débauche de couleurs et de formes, c'est précisément pour rappeler dans le monde entier que les corps sont traversés par les rapports socio-sexuels, pour rappeler aussi que la jouissance est politique, pour réconcilier enfin la politique avec le plaisir, c'est-à-dire en dernière analyse, avec le corps » (Tin, 2018, p. 236).

\section{Bibliographie}

AMOSSY, R. (2006). L'argumentation dans le discours. Paris : Armand Colin.

Bernard, A. \& BOËTSCH, G. (2018). Dictionnaire du corps. Paris : CNRS Éditions.

BiALAS, M. (2020). Le discours de Nicolas Sarkozy. Rhétorique et mise en scène. Paris : L'Harmattan.

LE BRETON, D. (2017). Anthropologie du corps et modernité. Paris : Puf.

Charaudeau, P. (2005). Le discours politique. Les masques du pouvoir. Paris : Vuibert.

DEPECKER, L. (1995). Dictionnaire du français des métiers. Paris : Éditions du Sevil.

GOUDAILLIER, J-P. \& KACPRZAK, A. (2014). Fonctions identitaires en situations diglossiques:

argots - dialectes - patois. Łódź : Wydawnictwo Uniwersytetu Łódzkiego.

LACAN, J. (1971-1972). Le Séminaire, livre XIX, « Ou pire ». Paris : A. Colin.

MARZANO, M. (2017). Dictionnaire du corps. Paris : Quadrige/Puf.

PAVEAU, M.-A. (2014). Le discours pornographique. Paris : La Musardine. 
PAVEAU, M.-A. \& Perea, F. (2014). Un objet de discours pour les études pornographiques.

Questions de communication, 26, pp. 7-15.

RobRIEUX, J-J. (2000). Rhétorique et argumentation. Paris : Nathan.

TIN, L-G. (2018). Gai. In ANDrieu, B. \& BOËTSCH, G. (ed.), Dictionnaire du corps. Paris : CNRS Éditions, pp. 233-237.

\section{Sitographie}

https://www.collinsdictionary.com/ [27/05/2021].

https://www.larousse.fr/ [15/09/2020]. 